\title{
An Unusual Case of Suspected Microvascular Angina in a Newborn
}

\author{
Stefania Cataldo, ${ }^{1}$ Giuseppe Alberto Annoni, ${ }^{1}$ Stefano Fiocchi, ${ }^{2}$ Luigi Daniele Mauri, \\ Alessandra Corato, ${ }^{1}$ and Gabriele Vignati ${ }^{1}$ \\ ${ }^{1}$ Pediatric Cardiology Unit, Ospedale Niguarda Ca' Granda, Piazza Ospedale Maggiore 3, 20162 Milan, Italy \\ ${ }^{2}$ Neonatology Unit, Ospedale Niguarda Ca' Granda, Piazza Ospedale Maggiore 3, 20162 Milan, Italy \\ Correspondence should be addressed to Stefania Cataldo, stefania.cataldo@ospedaleniguarda.it
}

Received 29 June 2012; Accepted 30 September 2012

Academic Editors: P. Pavone and S. Tay

Copyright ( $) 2012$ Stefania Cataldo et al. This is an open access article distributed under the Creative Commons Attribution License, which permits unrestricted use, distribution, and reproduction in any medium, provided the original work is properly cited.

\begin{abstract}
Myocardial ischemia in pediatric population is uncommon and usually due to congenital heart disease or extracardiac conditions leading to poor coronary perfusion. A 6-day-old newborn presented with respiratory distress and signs of heart failure. ECG, echocardiography, and laboratory results were consistent with myocardial ischemia. Coronary angiography was performed to exclude anomalous origin of coronary arteries, showing normal coronary artery origin and course. Thrombophilia and extracardiac causes were ruled out. Clinical conditions improved with mechanical ventilation and diuretics, enzyme levels lowered, repolarisation and systolic function abnormalities regressed, but ischemic electrocardiographic and echocardiographic signs still presented during intense crying. Becaues of suspicion of microvascular angina, therapy with ASA and beta-blocker was started. At 5 month followup, the baby was in good clinical condition and no more episodes were recorded. We believe it is an interesting case, as no similar cases have been recorded till now.
\end{abstract}

\section{Introduction}

Myocardial ischemia is a rare condition in pediatric population. Neonatal myocardial ischemia has been reported in patients with congenital heart disease (especially anomalous origin of the left coronary artery from the pulmonary artery, left ventricular hypertrophy, pulmonary atresia with intact ventricular septum, truncus arteriosus, and great vessels transposition) and in extra-cardiac conditions leading to poor coronary perfusion (severe hypoxia, disseminated intravascular coagulopathy, renal artery thrombosis, and sepsis); rare causes include endocardial fibroelastosis and mediocalcinosis of the coronary arteries. In older children Kawasaki disease and congenital heart disease (including postsurgical conditions) are the main causes, together with cardiomyopathies, substance abuse, and genetic disorders [ 1 , 2]. Microvascular angina is a heterogeneous entity defined as typical angina-like chest pain with electrocardiographic and metabolic evidence of myocardial ischemia but normal coronary arteries on angiography. Multiple pathophysiologic mechanisms have been described, including inflammation, estrogen deficiency, insulin resistance, enhanced pain perception due to hyperreactivity of cardiac pain receptors, and/or abnormalities in pain signal's transmission or modulation at subcortical level, but microvascular dysfunction is the most likely $[3,4]$. We report a case of suspected microvascular angina in a newborn.

\section{Case Report}

A term female newborn, weighing $3.700 \mathrm{~kg}$, was born vaginally without any prenatal complications. Family history was negative for heart disease. Due to the finding of a heart murmur, transthoracic echocardiography (TTE) was performed before discharge: a moderate tricuspid regurgitation due to valvular dysplasia was found, while all other findings were normal. The baby was discharged in good general condition with indication for clinical followup. On the 6th day of life she was admitted to ER because of tachypnea, sweating, and failure to thrive. Body weight was $3.220 \mathrm{~kg}$. 


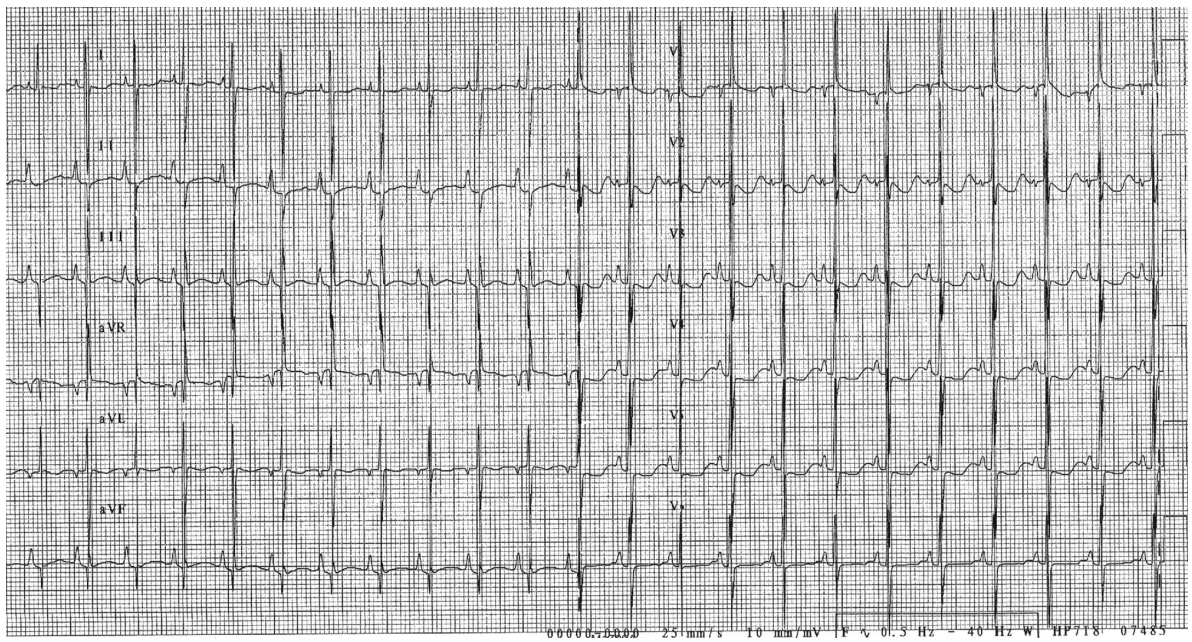

Figure 1

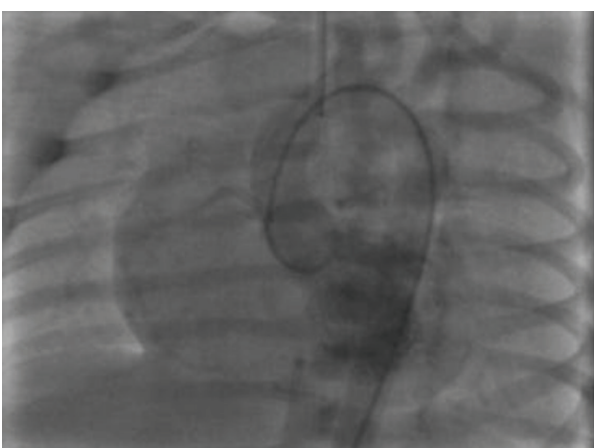

FIGURE 2

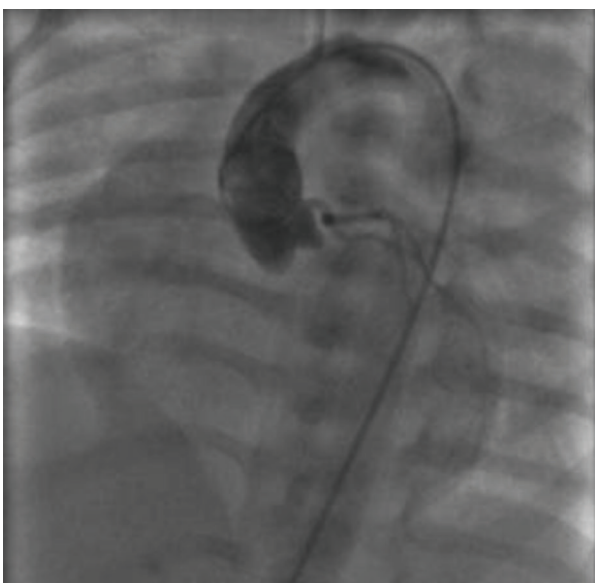

FIgURe 3

Signs of heart failure and respiratory distress were present: $\mathrm{O}_{2}$ saturation was $96 \%$ with $6 \mathrm{l} / \mathrm{min} \mathrm{O}_{2}$, venous blood gas analysis showed metabolic acidosis $\left(\mathrm{pH} 7.03, \mathrm{pO}_{2} 46 \mathrm{mmHg}\right.$, $\left.\mathrm{pCO}_{2} 29 \mathrm{mmHg}, \mathrm{HCO}_{3}-12.3 \mathrm{mmol} / \mathrm{L}, \mathrm{BE}-19\right)$. Mechanical ventilation was required and the patient was referred to our Department. Upon arrival, BP was $70 / 30 \mathrm{mmHg}$,
HR $130 \mathrm{bpm}$, and diuresis was preserved. Physical exam revealed a grade $3 / 6$ systolic murmur, weak femoral pulses, and liver was palpable $3 \mathrm{~cm}$ below costal arch. Laboratory studies were normal except for elevation of cardiac markers (high sensitivity troponin T $1001 \mathrm{ng} / \mathrm{L}, \mathrm{CK}-\mathrm{MB} 37.4 \mathrm{mcg} / \mathrm{L}$ ) and venous blood gas analysis $\left(\mathrm{pH}\right.$ 7.1, $\mathrm{pO}_{2}$ 65, $\mathrm{pCO}_{2}$ 28, Lac 14.8). Microbiological tests were negative for viral infection and chest radiograph showed cardiac enlargement and mild pulmonary venous congestion. ECG showed sinus rhythm 133 beats/min, right atrial enlargement, inferior Q waves, and lateral repolarisation abnormalities (Figure 1). At TTE evaluation left ventricle resulted of normal size, with hypokinetic inferior wall and dyskinetic septum and mildly depressed ejection fraction and the right ventricle was enlarged with mildly impaired systolic function. Moderate mitral regurgitation and severe tricuspid regurgitation were present. Right coronary artery (RCA) had a normal origin while it was difficult to visualize left coronary artery (LCA). To this purpose selective coronary angiography was performed; a normal origin and anatomy of RCA and LCA were confirmed (Figures 2 and 3) and all hemodynamic parameters resulted normal, too. Left ventriculography was not performed. With mechanical ventilation and diuretic therapy hemodynamics had improved and heart failure resolved. In a few hours the baby was successfully weaned from mechanical ventilation and ECG had normalized, except for long corrected QT interval (480 msec). Cardiac necrosis markers had progressively lowered (HS TnT $163.2 \mathrm{ng} / \mathrm{L}, \mathrm{CK}-\mathrm{MB}$ $5 \mathrm{mcg} / \mathrm{L})$. TTE revealed normal biventricular systolic function, absence of wall motion abnormalities, moderate tricuspid regurgitation, and no mitral regurgitation. Antithrombin III, protein C, and protein S levels were found to be normal. Ammonium, acylcarnitine panel, and plasma aminoacids levels were normal, too; urinary excretion of lactate and pyruvate was mildly elevated $(40 \mathrm{mM} / \mathrm{M}$ creatinine and $59 \mathrm{mM} / \mathrm{M}$ creatinine, resp.). Diuretics were gradually discontinued. Weight increased and suction was valid, but the baby still presented sudden crisis of sweating and crying, especially during feeding or stress (i.e., diagnostic examinations, 


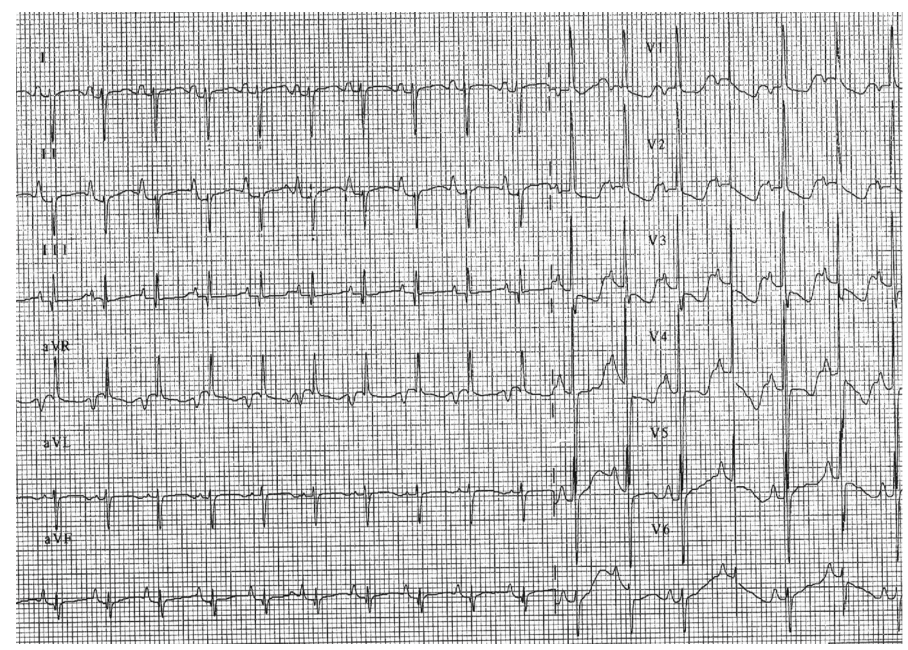

Figure 4

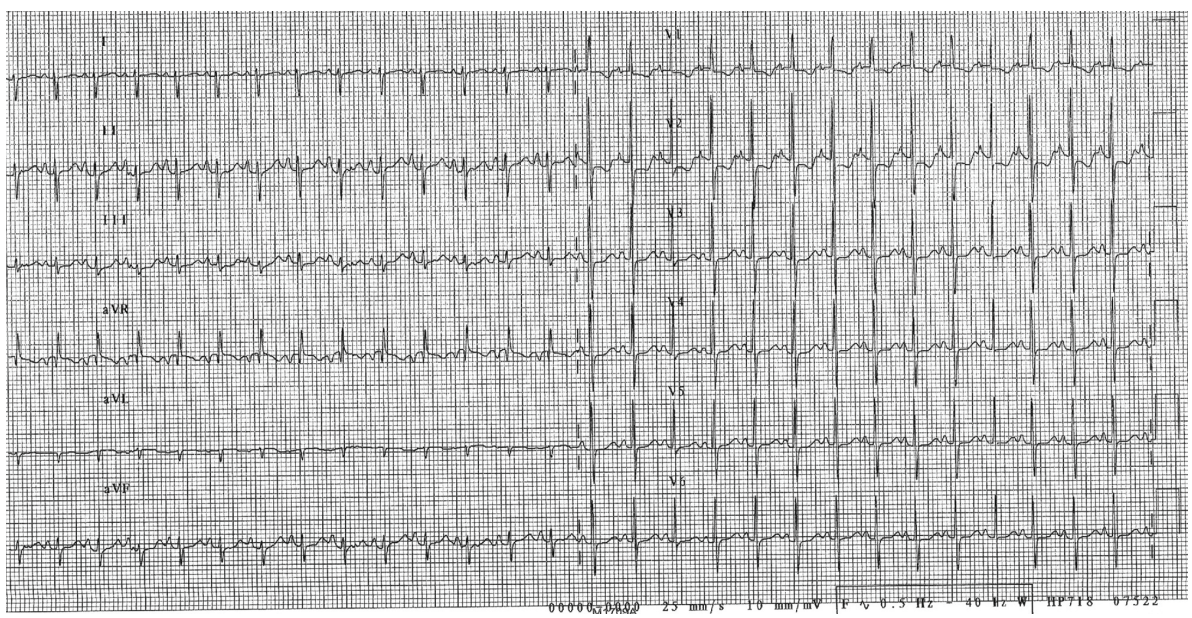

Figure 5

nursing care) lasting few minutes. ECG and TTE performed during one of these episodes showed a pseudonormalization of T waves followed by QRS axis rotation, lowering of ST segment in V2-V3, and severe reduction of left ventricular ejection fraction (25\%) (Figure 4). Therapy with atenolol (1 mg/kg), captopril (1 mg/kg), and aspirin $(3.5 \mathrm{mg} / \mathrm{kg})$ was started. During followup Holter ECG monitoring did not detect repolarization abnormalities and/or arrhythmias. Rest TTE after 20 days of therapy showed left ventricle ejection fraction 50\% with mild inferior wall motion reduction and mild tricuspid regurgitation. The baby was discharged at 40 days of life, weighing kg 4.120. Polygraphic sleep monitor was prescribed. At 5-month followup the baby was in good general condition, weighing $6 \mathrm{~kg}$ and there were no signs of heart failure. ECG showed normal repolarisation and corrected QT interval of $480 \mathrm{msec}$ (Figure 5).

\section{Discussion}

In pediatric patients myocardial ischemia can occur as unexplained sudden death or may present with weakness, irritability, loss of consciousness, and signs of poor cardiac output. Older children can also refer chest pain, palpitation, and dyspnea. ECG manifestations do not differ much from adults', but it is often not easy to differentiate between ischemic ECG and normal variants and/or ECG abnormalities associated with congenital heart disease. Electrocardiographic criteria to diagnose myocardial ischemia in children have been published (wide Q waves $(>35 \mathrm{~mm}$ ) with or without Q-wave notching, ST segment elevation ( $>2 \mathrm{~mm}$ ), and prolonged corrected QT interval (>440 msec) with Q-wave abnormalities [5]), but their reliability in prospective clinical use is not known. Concerning laboratory studies, there is little evidence on the reliability of enzymes' dosage for evaluation of pediatric patients with suspected myocardial ischemia. Neonatal myocardial ischemia has been described in cases of abnormal coronary arteries, congenital heart disease, thromboembolic events, and perinatal asphyxia. In the case presented, a newborn without congenital heart disease developed clinical, electrocardiographic, and echocardiographic signs of subepicardial damage of the lateral and inferior myocardial wall. On the basis of clinical 
presentation, differential diagnosis for myocardial ischemia was entertained, including congenital heart disease and extra-cardiac causes of reduced coronary perfusion. Angiography showed normal coronary artery origin, thus excluding the most frequent causes of ischemia in this particular population. Clinical findings were not consistent with sepsis and severe hypoxia, metabolic disorders and thrombophilia were ruled out, too. In the case presented, ischemia was induced by stress, as demonstrated by repolarisation and echocardiographic abnormalities during crying. On these bases, all findings were consistent with microvascular angina. Different therapeutic strategies have been proposed to treat adults with microvascular angina; among antianginal drugs, beta-blockers seem to be most effective and are considered first-line treatment [6]. Even if no cases of microvascular angina in pediatric age have been reported we decided to start beta-blocker administration in accordance to coexisting QTc prolongation. At 5 month followup no more episodes of MI were recorded. We believe that this diagnosis must be taken into account when facing clinical evidence of MI in presence of normal coronary angiography, even in small infants. It is a diagnosis per exclusionem, though, and more evidences are required to reach a better insight of this rare condition.

\section{Disclosure}

All authors listed have made contributions including collecting of data, reviewing literature, and conception. All of them gave an active contribution to drafting or revising this paper and none of them has conflict of interests to disclose. Moreover, they did not receive any financial support in the preparation of this paper.

\section{References}

[1] J. D. Reich and R. Campbell, "Myocardial infarction in children," The American Journal of Emergency Medicine, vol. 16, no. 3, pp. 296-303, 1998.

[2] D. S. Celermajer, G. F. Sholler, R. Howman-Giles et al., "Myocardial infarction in childhood: clinical analysis of 17 cases and medium term follow-up of survivors," British Heart Journal, vol. 65, no. 6, pp. 332-336, 1991.

[3] M. Singh, S. Singh, R. Arora et al., "Cardiac syndrome X:current concepts," International Journal of Cardiology, vol. 142, no. 2, pp. 113-119, 2010.

[4] S. D. Rosen, E. Paulesu, R. J. S. Wise et al., "Central neural contributing to the perception of chest pain in cardiac syndrome X," Heart, vol. 87, pp. 513-519, 2002.

[5] J. A. Towbin, J. Timothy Bricker, and A. Garson Jr., "Electrocardiographic criteria for diagnosis of acute myocardial infarction in childhood," The American Journal of Cardiology, vol. 69, no. 19, pp. 1545-1548, 1992.

[6] G. A. Lanza, G. Colonna, V. Pasceri et al., "Atenolol versus amlodipine versus isosorbide-5-mononitrate on anginal symptoms in syndrome X," The American Journal of Cardiology, vol. 84, no. 7, pp. 854-856, 1999. 


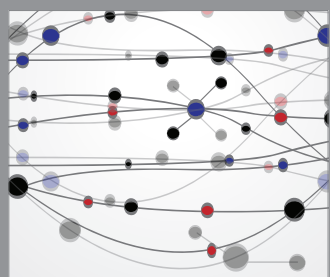

The Scientific World Journal
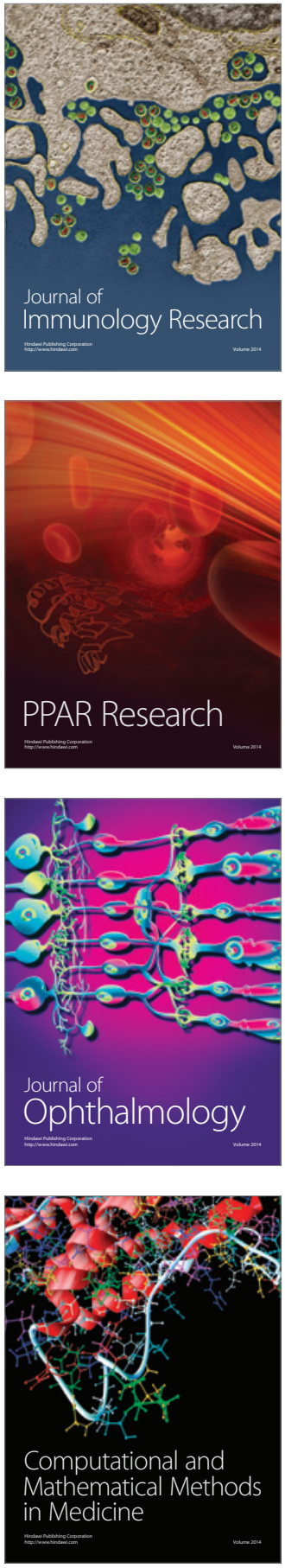

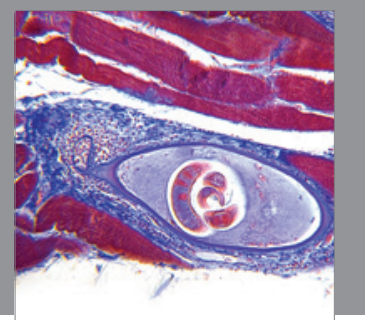

Gastroenterology

Research and Practice
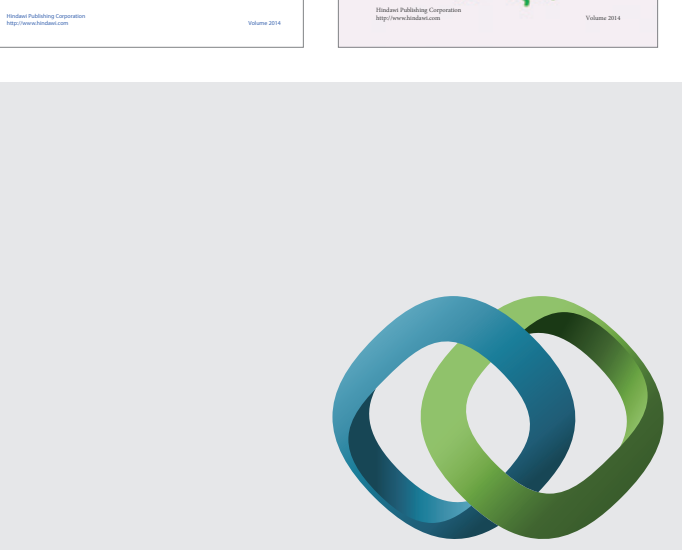

\section{Hindawi}

Submit your manuscripts at

http://www.hindawi.com
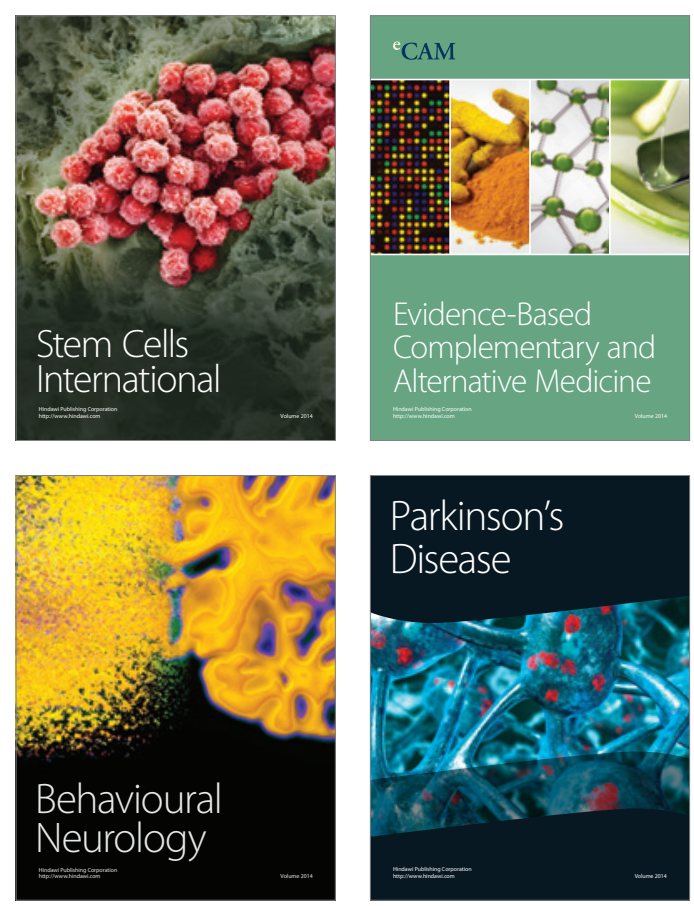

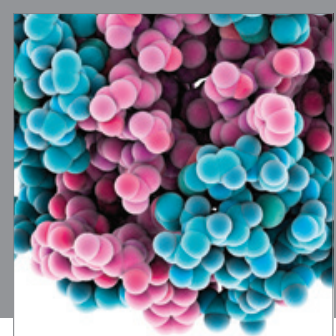

Journal of
Diabetes Research

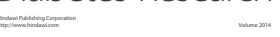

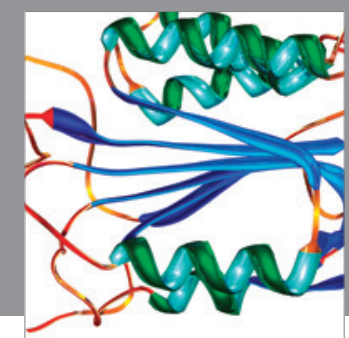

Disease Markers
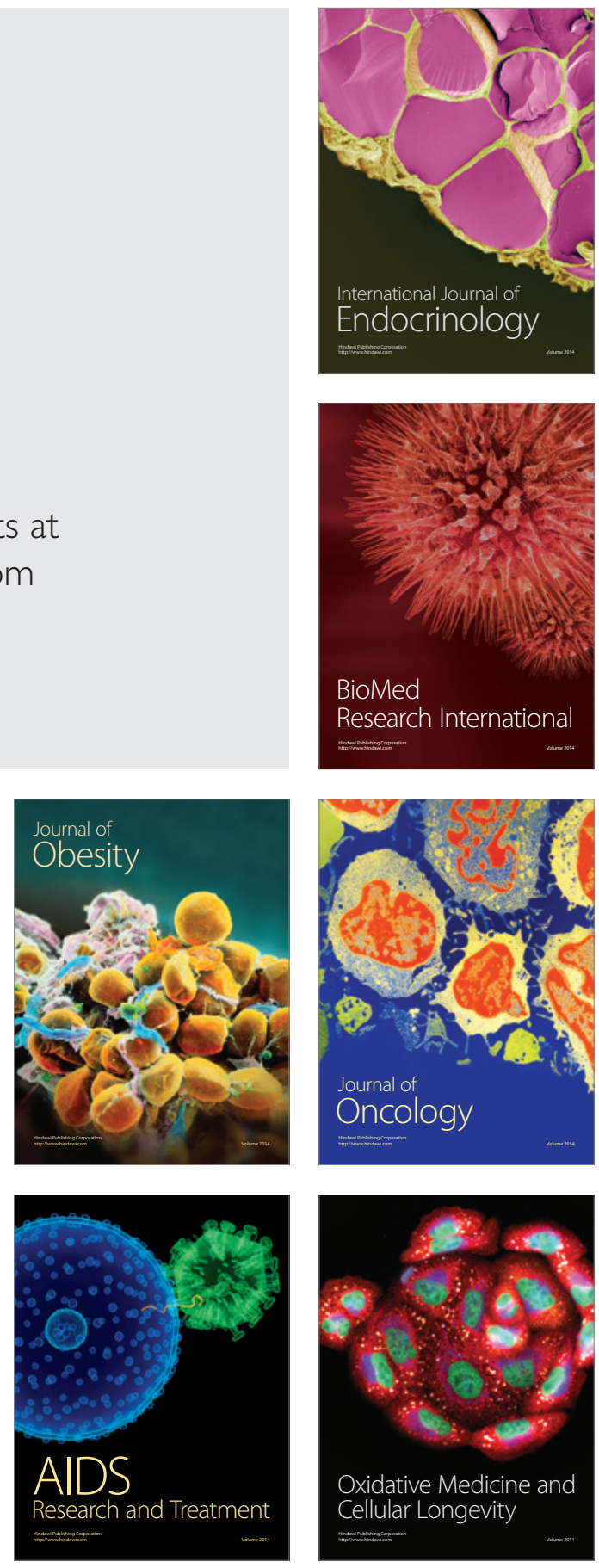